\title{
Measuring the electrical properties of semiconductor nanowires using terahertz conductivity spectroscopy
}

\author{
Hannah J. Joyce*a,b $^{\text {a,b }}$ Callum J. Docherty ${ }^{\mathrm{a}}$, Chaw-Keong Yong ${ }^{\mathrm{a}}$, Jennifer Wong-Leung ${ }^{\mathrm{c}}$, Qiang Gao ${ }^{\mathrm{c}}$, \\ Suriati Paiman $^{\mathrm{d}}$, H. Hoe Tan ${ }^{\mathrm{c}}$, Jagadish, C. ${ }^{\mathrm{c}}$, James Lloyd-Hughes ${ }^{\mathrm{e}}$, Laura M. Herz ${ }^{\mathrm{a}}$, and Michael B. \\ Johnston $^{\mathrm{a}}$ \\ ${ }^{\mathrm{a}}$ Department of Physics, University of Oxford, Clarendon Laboratory, Parks Road, \\ Oxford OX1 3PU, United Kingdom \\ ${ }^{\mathrm{b}}$ Department of Engineering, University of Cambridge, 9 JJ Thomson Avenue \\ Cambridge CB3 0FA, United Kingdom \\ ${ }^{\mathrm{c}}$ Department of Electronic Materials Engineering, Research School of Physics and Engineering, \\ The Australian National University, Canberra ACT 0200, Australia \\ ${ }^{\mathrm{d}}$ Department of Physics, Faculty of Science, Universiti Putra Malaysia, 43400 Serdang, Selangor, \\ Malaysia \\ ${ }^{e}$ Department of Physics, University of Warwick, Coventry, CV4 7AL, United Kingdom
}

\begin{abstract}
Accurately measuring the electronic properties of nanowires is a crucial step in the development of novel semiconductor nanowire-based devices. With this in mind, optical pump-terahertz probe (OPTP) spectroscopy is ideally suited to studies of nanowires: it provides non-contact measurement of carrier transport and dynamics at room temperature. OPTP spectroscopy has been used to assess key electrical properties, including carrier lifetime and carrier mobility, of GaAs, InAs and InP nanowires. The measurements revealed that InAs nanowires exhibited the highest mobilities and InP nanowires exhibited the lowest surface recombination velocity.
\end{abstract}

Keywords: Semiconductor nanowires, III-V nanowires, terahertz spectroscopy, surface recombination velocity, mobility, GaAs, InAs, InP.

\section{INTRODUCTION}

III-V nanowires, such as GaAs, InAs and InP nanowires, exhibit outstanding potential as nanocomponents for future electronic and optoelectronic devices. A wide variety of nanowire based devices have been demonstrated, including highly efficient solar cells, ${ }^{1,2}$ lasers ${ }^{3}$ and field-effect transistors for ultrahigh density logic and memory devices. ${ }^{4}$ Their unique geometry confers significant advantages over conventional planar heterostructures. If the nanowire diameter is sufficiently small, these structures can exhibit quantum confinement in two directions. Their high surface area to volume ratio makes nanowires ideal for sensing applications. Device architectures of vertical-standing nanowires will allow ultrahigh device density integration onto a single chip. Nanowires can be designed with axial and radial heterojunctions and homojunctions, not possible with conventional planar designs, and this greatly expands the range of device possibilities. Furthermore, nanowires can be grown epitaxially on Si without antiphase defects or misfit dislocations, which will enable monolithic integration of III-V nanowire optoelectronic devices with established Si microelectronics technology. ${ }^{5}$

*hannah.joyce@eng.cam.ac.uk; phone +44 1223748379

Micro/Nano Materials, Devices, and Systems, edited by James Friend, H. Hoe Tan, Proc. of SPIE Vol. 8923, 892321 - (c) 2013 SPIE · CCC code: 0277-786X/13/\$18 - doi: 10.1117/12.2049016 
A crucial step in the development of these novel nanowire-based devices is the accurate measurement of nanowire electronic properties. Unfortunately, measuring nanowire electrical properties using traditional contact-based techniques is challenging: forming electrical contacts to nanoscale structures is technically difficult and introduces artifacts. ${ }^{6}$

To avoid these problems, non-contact probes of nanowire conductivity are sought. Optical pump-terahertz probe (OPTP) spectroscopy is one such non-contact probe, and is sensitive to carrier transport and dynamics at room temperature. It is therefore ideally suited to studies of nanowires. Recent OPTP studies of Si, Ge and III-V nanowires are evidence of the strength of this technique. ${ }^{7-12}$

The electrical properties of GaAs, InAs and InP nanowires have been measured using OPTP spectroscopy. This allowed measurement of carrier mobility, carrier lifetime, surface recombination velocity and doping concentration. InAs nanowires exhibited the highest electron mobilities above $5000 \mathrm{~cm}^{2} \mathrm{~V}^{-1} \mathrm{~s}^{-1} \cdot{ }^{13} \mathrm{InP}$ nanowires exhibited the longest photoconductivity lifetimes and an extremely low surface recombination velocity. ${ }^{11}$ GaAs nanowires exhibited the shortest photoconductivity lifetimes of less than $5 \mathrm{ps}$, and the highest surface recombination velocity. ${ }^{13}$

\section{EXPERIMENTAL DETAILS}

\subsection{Nanowire growth}

The development of III-V nanowire-based devices depends on the ability to fabricate nanowires with tight control over nanowire properties. The most common, and arguably the most promising growth technique is MOCVD using $\mathrm{Au}$ nanoparticles to drive nanowire growth. The Au nanoparticles collect reaction species and drive highly anisotropic nanowire growth at the nanoparticle-nanowire interface, as illustrated in Figure 1. This approach offers high flexibility and accuracy in nanowire design and is readily scalable for industrial mass fabrication. It achieves epitaxial nanowires that are free standing on the growth substrate and it offers a number of growth parameters which can be tailored for optimum nanowire growth, ${ }^{14}$ including temperature, V/III ratio and the absolute flow rates of group III and group V precursor species. For example, by choosing appropriate growth parameters, nanowire crystal structure can be tuned between zinc-blende and wurtzite, ${ }^{15}$ or their charge carrier lifetime and mobility can be enhanced ${ }^{10}$.

In this study, growths were performed in an AIXTRON 200/4 horizontal flow MOCVD reactor with $\mathrm{H}_{2}$ carrier gas at a total gas flow rate of $15 \mathrm{slm}$ and a pressure of $100 \mathrm{mbar}$. The group III precursors were TMGa, TMIn and TMAl, and the group $\mathrm{V}$ precursors were $\mathrm{AsH}_{3}$ and $\mathrm{PH}_{3}$. The nanowires were grown on epi-ready (111)B oriented GaAs, InAs and InP substrates, with substrate material chosen to be the same as the nanowire material, for example GaAs nanowires were grown on GaAs (111)B substrates.

Before growth, the substrates were functionalised with $0.1 \%$ poly-L-lysine solution and then treated with a solution of colloidal $\mathrm{Au}$ nanoparticles of a particular diameter $(20,30,50$ or $80 \mathrm{~nm})$. Prior to growth initiation the substrate was annealed under group $\mathrm{V}$ pressure to desorb surface contaminants. GaAs nanowires were grown using a two-temperature procedure as published previously. ${ }^{16}$ InAs nanowires were grown at $400^{\circ} \mathrm{C}$ and a V/III ratio of 46 to yield zinc-blende crystal structure ${ }^{15}$ For InP nanowires, growth was performed at $420{ }^{\circ} \mathrm{C}$ for 20 minutes using TMIn and $\mathrm{PH}_{3}$ precursors with a V/III ratio of $700 .{ }^{17}$

Field-emission scanning electron microscopy (FESEM) and transmission electron microscopy (TEM) were used to determine nanowire morphology and crystal structure, respectively. FESEM was carried out using a Hitachi S4300 at an accelerating voltage of $5 \mathrm{kV}$. For TEM investigations, nanowires were mechanically transferred to holey carbon grids and TEM was performed using a Phillips CM300 operated at $300 \mathrm{kV}$. Photoluminescence (PL), time-resolved

photoluminescence (TRPL) and terahertz conductivity spectroscopy were used to examine the charge carrier dynamics and transport in these nanowires. Experimental details for PL and TRPL are described elsewhere. ${ }^{11}$ 


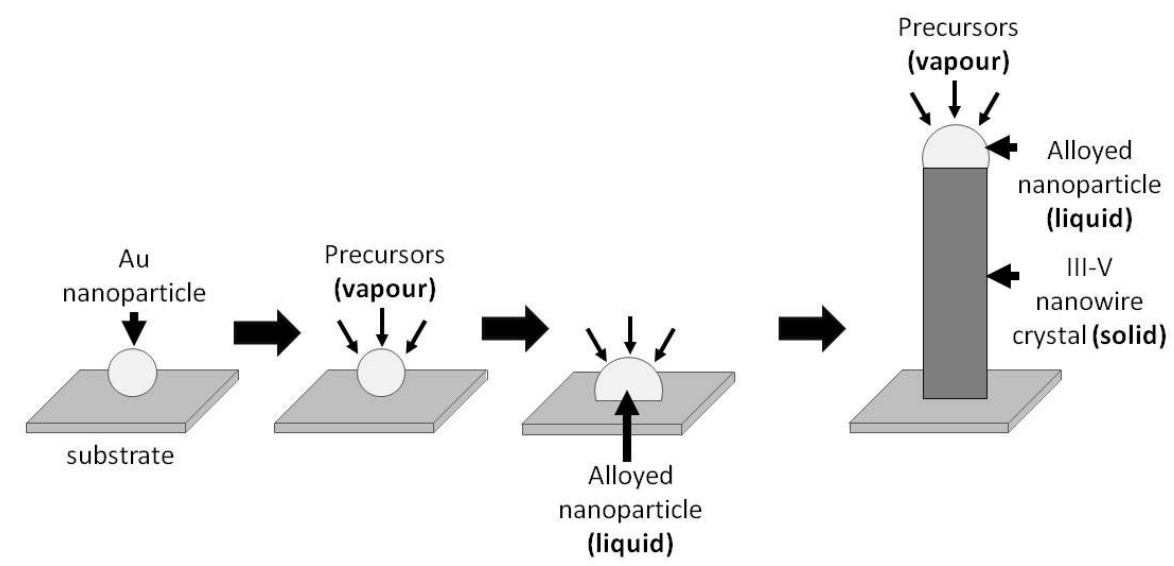

Figure 1. Stages of nanowire growth by the vapour-liquid-solid growth mechanism. Au nanoparticles drive nanowire growth. Group III (Ga and In) and Group V (As and P) species are supplied from the vapour phase. At growth temperature the nanoparticle forms a eutectic liquid alloy with the group III species. The III-V crystal grows at the interface between the nanoparticle and the underlying semiconductor. Thus, the Au nanoparticle drives highly anisotropic nanowire growth. The process is termed the vapour-liquid-solid mechanism because it involves vapour, liquid and solid phases: vapour phase precursors, a eutectic liquid alloy nanoparticle, and a solid nanowire.

\subsection{Terahertz time-domain spectroscopy}

After growth, nanowires were transferred to z-cut quartz by rubbing the as-grown substrates against the quartz substrates. The quartz substrates are suitable as they are highly transparent to $\mathrm{THz}$ radiation and do not exhibit any photoconductivity response.

Terahertz generation, photoexcitation of the sample, and terahertz detection were all performed using an amplified Ti:Sapphire laser generating $35 \mathrm{fs}$ pulses centred at $800 \mathrm{~nm}$ at a $5 \mathrm{kHz}$ repetition rate and $4 \mathrm{~W}$ average power. Each laser pulse was split into three paths: (i) $200 \mathrm{~mJ} /$ pulse was used to generate the $\mathrm{THz}$ probe pulse via optical rectification in a $2 \mathrm{~mm} \mathrm{GaP}$ crystal, (ii) $1.6 \mathrm{~mJ} /$ pulse was used as a gate for electro-optic detection of the transmitted $\mathrm{THz}$ pulse with a 200 $\mathrm{mm} \mathrm{GaP}$ crystal, and (iii) the remainder was used as the optical pump to photoexcite the sample. The optical pump beam was attenuated using neutral density filters to produce sample photoexcitation fluences between 1 and $160 \mathrm{~mJ} / \mathrm{cm}^{2}$. At the sample position, the optical pump beamwidth had a full width at half maximum (FWHM) of $13 \mathrm{~mm}$, whereas the $\mathrm{THz}$ probe FWHM was only $1.3 \mathrm{~mm}$. Consequently, the terahertz probe measured an area of approximately constant photoexcited carrier density. The THz electric field, $E$, was detected using a balanced photodiode circuit connected to a lock-in amplifier referenced to a $2.5 \mathrm{kHz}$ chopper in the THz generation beam. A second lock-in amplifier was used to detect the photoinduced change in terahertz electric field, $\Delta E$, by referencing to a $125 \mathrm{~Hz}$ chopper in the optical pump beam. To investigate the THz spectral response of the material as a function of time after photoexcitation, we varied the delay between the optical pump, terahertz probe and optical gate pulse. The measurements were performed at room temperature. The entire terahertz beam path was kept under vacuum to avoid absorption of the terahertz radiation by atmospheric water vapour.

\section{RESULTS AND DISCUSSION}

\subsection{Photoconductivity decay}

Nanowires of different diameters, $d$, were investigated. Figure 2 (a and b) shows SEM images of InAs nanowires of different diameters. Their photoconductivity decay curves are plotted in Figure 2c. The decay curves were well fitted by single exponential functions with lifetimes $\tau$. As seen in Figure 2c, narrow diameter InAs nanowires exhibit shorter carrier lifetimes than wider diameter nanowires. This is a consequence of surface recombination, which is more significant for narrow diameter nanowires with their higher surface area-to-volume ratios. The photoconductivity lifetime and nanowire diameter are related according to the formula 


$$
\frac{1}{\tau}=\frac{1}{\tau_{v}}+\frac{4 S}{d}
$$

where $\tau_{\mathrm{v}}$ is the lifetime in bulk material and $S$ is the surface recombination velocity. By fitting Equation (1) to the lifetime data, the surface recombination velocity can be extracted, as shown in Figure $2 \mathrm{~d}$. The surface recombination velocities of these InAs nanowires was calculated as $3.0 \times 10^{3} \mathrm{~cm} / \mathrm{s} .{ }^{13}$
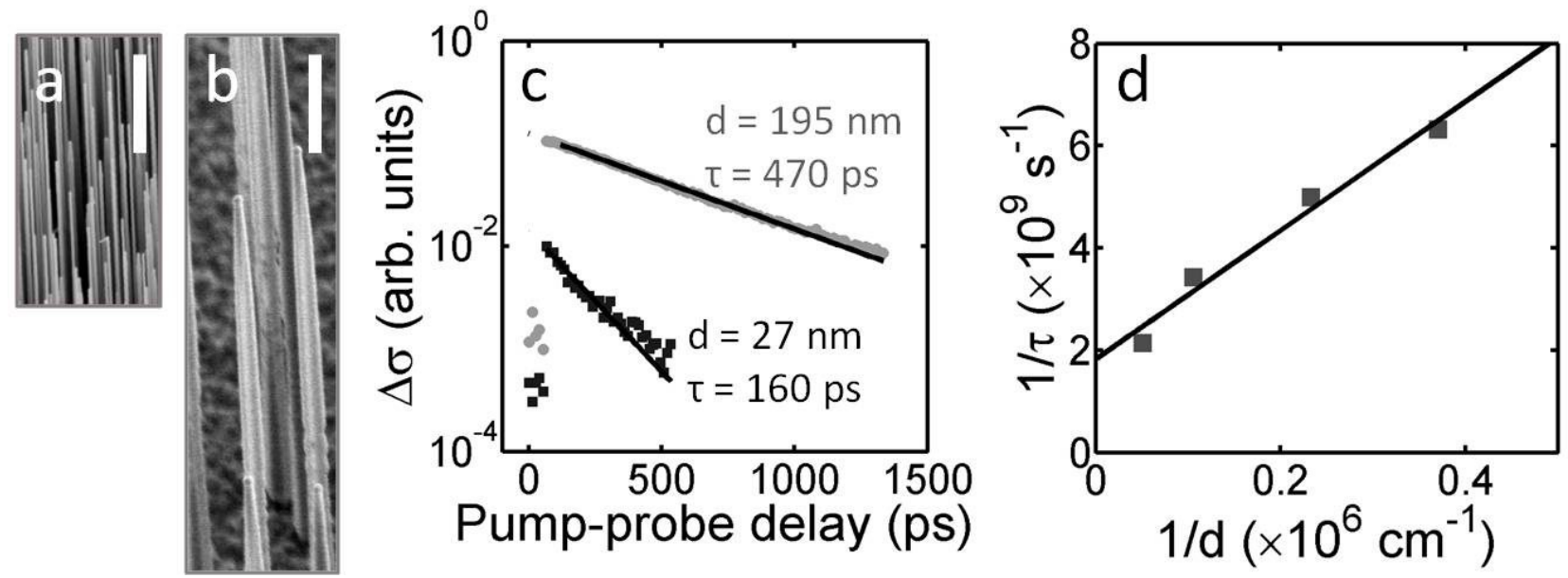

Figure 2: Results for InAs nanowires. SEM images of InAs nanowires with average diameters of (a) $27 \mathrm{~nm}$, and (b) $195 \mathrm{~nm}$. Scale bars are $1 \mu \mathrm{m}$. Samples were tilted at $40^{\circ}$. (c) Photoconductivity decays for these nanowires, showing fitted lifetimes, $\tau$. (d) Plot of decay rate $(1 / \tau)$ versus inverse nanowire diameter $(1 / d)$ for four different diameters (squares). The slope of the fit (solid line) is used to determine the surface recombination velocity, $S$.

Photoconductivity decay curves were also measured for GaAs and InP nanowires of varying diameters. Using the same process outlined above for InAs nanowires, surface recombination velocities were calculated for GaAs and InP nanowires. ${ }^{11,13}$ GaAs nanowires featured the shortest photoconductivity lifetimes of only 1 to $5 \mathrm{ps,} \mathrm{and} \mathrm{exhibited} \mathrm{the}$ highest surface recombination velocity of $5.4 \times 10^{5} \mathrm{~cm} / \mathrm{s} .{ }^{13}$ This suggests their potential for ultrafast electronic switching devices. Furthermore, it indicates that surface passivation is essential to increase carrier lifetimes in GaAs nanowires. ${ }^{10,18}$ In marked contrast, InP nanowires exhibited the longest photoconductivity lifetimes of over $1 \mathrm{~ns}$ and the lifetimes showed only a weak dependence on nanowire diameter: nanowires of $50 \mathrm{~nm}$ diameter exhibited $1.2 \mathrm{~ns}$ lifetimes whereas nanowires of $160 \mathrm{~nm}$ diameter exhibited marginally longer lifetimes of $1.4 \mathrm{~ns}$. This is related to the extremely low surface recombination velocity of InP nanowires, which was measured as $170 \mathrm{~cm} / \mathrm{s}$. This prolonged photoconductivity and low surface recombination velocity of InP nanowires is promising for many electronic applications which require long carrier lifetimes, including photovoltaics.

\subsection{Photoconductivity spectra}

Photoconductivity spectra of all samples exhibited a pronounced Lorentzian response. An example of this response is shown in Figure 3 for InAs nanowires. The resonant frequency increased with photoexcited carrier density. This type or response is characteristic of localised surface plasmon modes, which typically occur in the terahertz range for semiconductors nanostructures. ${ }^{13}$ To these spectra we fitted Lorentzian functions as detailed in previous publications. ${ }^{11,13}$ The electron scattering rate, $\gamma$, is a fitting parameter, which can readily be converted to electron mobility via

$$
\mu=\frac{q}{m_{e}^{*} \gamma}
$$


where $m_{e}{ }^{*}$ is the electron effective mass and $q$ is the electronic charge. Thus, the fits allowed extraction of electron mobility for each sample. InAs nanowires exhibited very high electron mobilities of $6000 \mathrm{~cm}^{2} \mathrm{~V}^{-1} \mathrm{~s}^{-1} .{ }^{13}$ This suggests their potential for high mobility applications such as in field effect transistors. InP nanowires exhibited the lowest electron mobilities of only 200 to $600 \mathrm{~cm}^{2} \mathrm{~V}^{-1} \mathrm{~s}^{-1}{ }^{11}$ This value is significantly lower than typical values for bulk InP. This observation, together with modelling, indicates the carrier mobility in InP nanowires is strongly degraded by the high density of crystallographic stacking faults in these nanowires. ${ }^{11}$ This points to the importance of engineering InP nanowires with high crystal quality for future device applications.

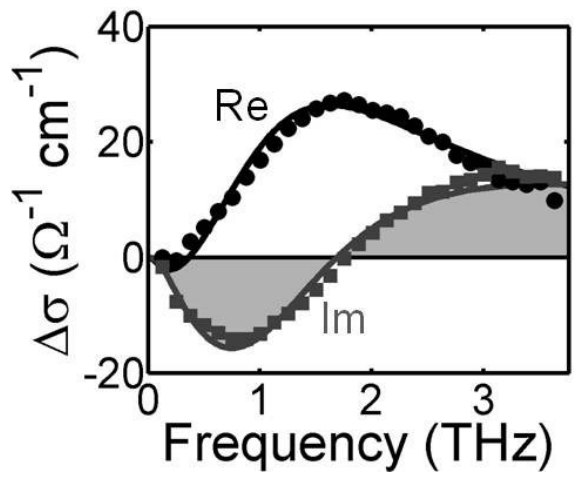

Figure 3: Photoconductivity spectrum for InAs nanowires of $45 \mathrm{~nm}$ diameter. The solid symbols are the measured data and the lines show the Lorentzian fit. The real part of the photoconductivity is shown in black and the imaginary part is shown in grey.

\section{CONCLUSIONS}

The properties of various III-V nanowires were investigated with OPTP spectroscopy. InAs nanowires exhibited the highest electron mobilities above $5000 \mathrm{~cm}^{2} \mathrm{~V}^{-1} \mathrm{~s}^{-1}$ which indicates their suitability for high mobility devices. ${ }^{13}$ InP nanowires exhibited the longest photoconductivity lifetimes of over $1 \mathrm{~ns}$ and an extremely low surface recombination velocity. ${ }^{11}$ This makes InP nanowires promising for applications which require long carrier lifetimes, such as photovoltaics. These findings will guide the choice of nanowires for different applications and enable nanowire growers to optimise material quality for device applications.

\section{ACKNOWLEDGEMENTS}

The authors acknowledge financial support from the Engineering and Physical Sciences Research Council (UK), the Australian Research Council, and the Australian National Fabrication Facility.

\section{REFERENCES}

[1] Wallentin, J., Anttu, N., Asoli, D., Huffman, M., Aberg, I., Magnusson, M. H., Siefer, G., Fuss-Kailuweit, P., Dimroth, F., et al., "InP nanowire array solar cells achieving 13.8\% efficiency by exceeding the ray optics limit.," Science 339, 1057-1060 (2013) [doi:10.1126/science.1230969].

[2] Krogstrup, P., Jørgensen, H. I., Heiss, M., Demichel, O., Holm, J. V., M. Aagesen, J. Nygard, and A. Fontcuberta, "Single-nanowire solar cells beyond the Shockley-Queisser limit," Nat. Photonics 7, 306-310, (2013) [doi:10.1038/NPHOTON.2013.32].

[3] Saxena, D., Mokkapati, S., and Jagadish, C., "Semiconductor Nanolasers," IEEE Photonics J. 4, 582-585 (2012) [doi:10.1109/JPHOT.2012.2189201].

[4] Tomioka, K., Yoshimura, M., and Fukui, T., "A III-V nanowire channel on silicon for high-performance vertical transistors.," Nature 488, 189-192 (2012) [doi:10.1038/nature11293]. 
[5] Kang, J., Gao, Q., Joyce, H. J., Tan, H. H., Jagadish, C., Kim, Y., Guo, Y., Xu, H., Zou, J, Fickenscher, M. A. Smith, L. M., Jackson, H. E., and Yarrison-Rice, J. M., "Defect-free GaAs/AlGaAs core-shell nanowires on Si substrates," Cryst. Growth Des. 11, 3109-3114 (2011).

[6] Dayeh, S., Soci, C., and Yu, P., "Transport properties of InAs nanowire field effect transistors: The effects of surface states," J. Vac. Sci. Technol. B Microelectron. Nanom. Struct. 25(4), 1432-1436 (2007) [doi:10.1116/1.2748410].

[7] Ulbricht, R., Kurstjens, R., and Bonn, M., "Assessing Charge Carrier Trapping in Silicon Nanowires Using Picosecond Conductivity Measurements," Nano Lett. 12, 3821-3827 (2012).

[8] Strait, J. and George, P., "Measurements of the carrier dynamics and terahertz response of oriented germanium nanowires using optical-pump terahertz-probe spectroscopy," Nano Lett. 9, 2967-2972 (2009).

[9] Parkinson, P, Lloyd-Hughes, J., and Gao, Q., “Transient terahertz conductivity of GaAs nanowires," Nano Lett. 7(7), 2162-2165 (2007).

[10] Parkinson, P, Joyce, H. J., Gao, Q., Tan, H. H., X. Zhang, Zou, J, Jagadish, C., Herz, L. M., and Johnston, M. B., "Carrier lifetime and mobility enhancement in nearly defect-free core- shell nanowires measured using timeresolved terahertz spectroscopy," Nano Lett. 9(9), 3349-3353 (2009).

[11] Joyce, H. J., J. Wong-Leung, Yong, C.-K., Docherty, C. J., Paiman, S., Gao, Q., Tan, H. H., Jagadish, C., LloydHughes, J., Herz, L. M., and Johnston, M. B., "Ultralow surface recombination velocity in InP nanowires probed by terahertz spectroscopy.," Nano Lett. 12(10), 5325-5330 (2012) [doi:10.1021/nl3026828].

[12] Parkinson, P, C. Dodson, Joyce, H. J., K. A. Bertness, N. A. Sanford, Herz, L. M., and Johnston, M. B., "Noncontact measurement of charge carrier lifetime and mobility in GaN nanowires.," Nano Lett. 12(9), 46004604 (2012) [doi:10.1021/nl301898m].

[13] Joyce, H. J., C. J. Docherty, Gao, Q., Tan, H. H., Jagadish, C., Lloyd-Hughes, J., Herz, L. M., and Johnston, M. B., "Electronic properties of GaAs, InAs and InP nanowires studied by terahertz spectroscopy.," Nanotechnology 24(21), 214006 (2013) [doi:10.1088/0957-4484/24/21/214006].

[14] Joyce, H. J., Gao, Q., Tan, H. H., Jagadish, C., Kim, Y., Zou, J, Smith, L. M., Jackson, H. E., Yarrison-Rice, J. M., Parkinson, P, and Johnston, M. B., "III - V semiconductor nanowires for optoelectronic device applications," Prog. Quantum Electron. 35, 23-75 (2011) [doi:10.1016/j.pquantelec.2011.03.002].

[15] Joyce, H. J., J. Wong-Leung, Gao, Q., Tan, H. H., and Jagadish, C., "Phase perfection in zinc blende and wurtzite III-V nanowires using basic growth parameters.," Nano Lett. 10(3), 908-915 (2010) [doi:10.1021/n1903688v].

[16] Joyce, H. J., Gao, Q., Tan, H. H., Jagadish, C., Kim, Y., Zhang, X., Guo, Y., and Zou, J, “Twin-free uniform epitaxial GaAs nanowires grown by a two-temperature process.," Nano Lett. 7(4), 921-926 (2007) [doi:10.1021/nl062755v].

[17] Paiman, S., Gao, Q., Joyce, H. J., Kim, Y., Jagadish, C., Zhang, X., Guo, Y., and Zou, J, "Growth temperature and V/III ratio effects on the morphology and crystal structure of InP nanowires," J. Phys. D ... 43(44), 445402 (2010) [doi:10.1088/0022-3727/43/44/445402].

[18] Titova, L. V., Hoang, T. B., H. E. Jackson, L. M. Smith, Kim, Y., Joyce, H. J. Tan, H. H., and Jagadish, C. "Temperature dependence of photoluminescence from single core-shell GaAs - AlGaAs nanowires," Appl. Phys. Lett. 89, 173126 (2006) [doi:10.1063/1.2364885]. 